\title{
Dermacoccus nishinomiyaensis
}

National Cancer Institute

\section{Source}

National Cancer Institute. Dermacoccus nishinomiyaensis. NCI Thesaurus. Code C86336.

A species of aerobic, Gram positive, cocci shaped bacterium in the phylum Actinobacteria.

This species is catalase, urease and oxidase positive, coagulase negative grows bright orange colonies in culture, does not hydrolyze esculin and is indole negative. D.

nishinomiyaensis is found in water and on the skin of mammals and is considered to be non-pathogenic. 